\title{
Efeito da temperatura sobre a composição físico-química e compostos bioativos de farinha de taro obtida em leito de jorro
}

\section{Effect of temperature on the physicochemical composition and bioactive compounds of taro flour obtained in a spouted bed}

\section{Deise Souza de Castro ${ }^{1 *}$, Tharcia Kiara Beserra de Oliveira', Danielle Martins Lemos', Ana Paula Trindade Rocha ${ }^{2}$, Renata Duarte Almeida ${ }^{3}$}

\author{
1 Universidade Federal de Campina Grande (UFCG), Unidade Acadêmica de Engenharia Agrícola, Programa de Pós-graduação em Engenharia \\ Agrícola, Campina Grande/PB - Brasil \\ 2 Universidade Federal de Campina Grande (UFCG), Unidade Acadêmica de Engenharia de Alimentos, Centro de Tecnologia e Recursos Naturais, \\ Campina Grande/PB - Brasil \\ ${ }^{3}$ Universidade Federal de Campina Grande (UFCG), Centro de Ciência e Tecnologia, Programa de Pós-graduação em Engenharia de Processos, \\ Campina Grande/PB - Brasil
}

\section{${ }^{*}$ Corresponding Author}

Deise Souza de Castro, Universidade Federal de Campina Grande (UFCG), Unidade Acadêmica de Engenharia Agrícola, Rua Aprígio Veloso, 882 , Bodocongó, CEP: 58429140, Campina Grande/PB - Brasil, e-mail: deise_castro01@hotmail.com

Cite as: Effect of temperature on the physicochemical composition and bioactive compounds of taro flour obtained in a spouted bed. Braz. J. Food Technol., v. 20, e2016060, 2017.

Received: May 18, 2016; Accepted: Apr. 04, 2017

\section{Resumo}

A produção de farinhas a partir de frutas, hortaliças e de tubérculos não convencionais para esse processo vem sendo estudada como uma alternativa de produção de alimentos. Dentre os tubérculos, tem-se o taro, o qual pode ser consumido de várias maneiras: cozido, em forma de purê, como ingrediente para sopas e ensopados e em forma de farinha. Desta forma, objetivou-se com este trabalho estudar o processo de secagem por atomização do taro, em leito de jorro, sob diferentes temperaturas e avaliar a influência de condições operacionais no rendimento e na qualidade do produto após sua secagem. As análises físico-químicas realizadas no produto in natura e nas farinhas foram: teor de água, pH, acidez total titulável e ácido ascórbico total, atividade de água $\left(25^{\circ} \mathrm{C}\right)$ e cor, além de compostos fenólicos e flavonoides. As farinhas obtidas apresentaram vitamina $\mathrm{C}$ variando de 0,05 a 0,17 mg/100 g, compostos fenólicos variando de 4,42 a 5,60 mg/100 g e quantidade de flavonoides de 1,05 a 4,30 $\mu \mathrm{g} / 100 \mathrm{~g}$, tornando-as alimentos fonte de compostos bioativos, os quais podem ser adicionados na elaboração de outros produtos ou inseridos na dieta humana. Conclui-se que a farinha obtida sob a temperatura de $90^{\circ} \mathrm{C}$ apresentou os maiores conteúdos de vitamina $\mathrm{C}$, porém as farinhas obtidas sob as temperaturas de $70{ }^{\circ} \mathrm{C}$ e $80^{\circ} \mathrm{C}$ possuem $\mathrm{pH}$ próximo da neutralidade, além do teor de água em porcentagem reduzida prolongar a vida de prateleira do produto.

Palavras-chave: Colocasia esculenta; Secagem em leito de jorro; Farinha de inhame.

\section{Summary}

The production of flours from fruits, vegetables and not conventionally processed tubers, has been studied as alternative food production. Taro is one of these tubers, which can be consumed in various ways; cooked, pureed, as ingredients for soups and stews, and in the form of flours. Thus, the aim of this work was to study the drying process of taro by atomization in a spouted bed at different temperatures, and assess the influence of the operating conditions on the yield and quality of the product after drying. The following physicochemical analyzes were carried out on the fresh product and the meal: water content, $\mathrm{pH}$, titratable acidity, total ascorbic acid, water activity $\left(25^{\circ} \mathrm{C}\right)$ and colour, as well as the phenolic compound and flavonoid contents. The flours obtained showed amounts of vitamin $\mathrm{C}$ ranging from 0.05 to $0.17 \mathrm{mg} / 100 \mathrm{~g}$, phenolic compounds varying from 4.42 to $5.60 \mathrm{mg} / 100 \mathrm{~g}$, and flavonoids from 1.05 to $4.30 \mu \mathrm{g} / 100 \mathrm{~g}$ making them sources of bioactive compounds, which could be added in the preparation of other products or inserted into the human diet. 
It was concluded that the flour obtained at $90{ }^{\circ} \mathrm{C}$ showed the highest vitamin $\mathrm{C}$ content, but the flours obtained at temperatures of $70{ }^{\circ} \mathrm{C}$ and $80^{\circ} \mathrm{C}$ showed $\mathrm{pH}$ values near neutrality, besides a reduced water content favouring a longer storage life of the product.

Keywords: Colocasia esculenta; Spouted bed drying; Taro flour.

\section{Introdução}

A produção de farinhas a partir de frutas, hortaliças e tubérculos não convencionais ao beneficiamento de alimentos (SOUZA et al., 2012; BASSETTO et al., 2013) vem sendo estudada como alternativa de produção de alimentos que promovam benefícios nutricionais associados à manutenção da saúde.

A Colocasia esculenta é uma espécie conhecida popularmente como "taro" e pertence à família Araceae. Essa família é distribuída por todas as regiões tropicais, subtropicais e temperadas do mundo. Esse tubérculo pode ser consumido de várias maneiras: cozido, em forma de purê, como ingrediente para sopas e ensopados e na forma de farinha (TEIXEIRA et al., 2013).

A espécie Colocasia esculenta apresenta rizomas (parte comestível) que apresentam elevado valor nutritivo e energético, sendo excelente fonte de vitaminas do complexo B (tiamina, riboflavina e niacina), minerais (em destaque o ferro) e carboidratos (amido, principalmente), além de baixo teor de lipídios (SANTOS et al., 2007).

Embora os tubérculos em geral possuam alta qualidade nutricional e sejam muito apreciados na culinária brasileira, pouco tem sido observado no cultivo de $C$. esculenta. Essa espécie tem sofrido pouca investigação científica, pouco se sabe sobre suas técnicas de manejo, melhoramento genético, caracterização, potencial nutricional e industrial, como também não há relatos de estudos de secagem e armazenamento do produto (NASCIMENTO et al., 2015).

O amido, principal carboidrato encontrado nos tubérculos, é a mais importante fonte de hidratos de carbono em alimentos, representando cerca de $80 \%$ a $90 \%$ de todos os polissacáridos na dieta. É o principal responsável pelas propriedades tecnológicas que caracterizam muitos produtos alimentares e vem ganhando ainda mais atenção por causa de seus benefícios à saúde (BEZERRA et al., 2013).

Os tubérculos possuem uma grande facilidade para o escurecimento e, consequentemente, perda na qualidade do produto, com isso, o tempo entre a colheita e a industrialização deve ser curto. A secagem é uma excelente prática para manter o produto com características adequadas para o consumo por longos períodos. Na secagem em leito de jorro, a atomização da pasta ou suspensão sobre o leito reveste as partículas com uma fina camada de material. A película formada por meio de secagem se fragmenta devido aos efeitos de colisões entre partículas. É obtido um material na forma de pó, que é arrastado para fora pela corrente de ar e recolhido por um ciclone conectado ao secador. Desta forma, produz-se uma farinha de alta qualidade e baixo custo, preservando compostos bioativos, sendo possível realizar a secagem de materiais termossensíveis, oferecendo vantagens sobre os outros métodos de secagem, especialmente em relação ao tempo de secagem (BEZERRA et al., 2013; NASCIMENTO et al., 2015; COSTA et al., 2015).

Com isso, este trabalho teve como objetivo estudar o processo de secagem por atomização do taro, em leito de jorro, sob diferentes temperaturas e avaliar a influência de condições operacionais na qualidade do produto após sua secagem.

\section{Material e métodos}

O experimento foi realizado durante o mês de setembro de 2015 no Laboratório de Armazenamento e Processamento de Produtos Agrícolas, do Departamento de Engenharia Agrícola, da Universidade Federal de Campina Grande.

Os taros foram adquiridos na Central de Abastecimento - CEASA Recife, transportados em sacos de polietileno até o laboratório, onde foram lavados em solução de hipoclorito de sódio a 50 ppm, por 15 min e, posteriormente, enxaguados em água corrente. Depois da higienização, os inhames foram descascados e processados em centrífuga doméstica para obtenção do extrato líquido usado como suspensão (Figure 1). O extrato líquido foi submetido à secagem por atomização em leito de jorro, modelo FDB 1.0 (LABMAQ, Brasil), sob as temperaturas de $70^{\circ} \mathrm{C}, 80^{\circ} \mathrm{C}$ e $90^{\circ} \mathrm{C}$, vazão do ar no bico atomizador de $3,0 \mathrm{~m}^{3} / \mathrm{min}$ e vazão de alimentação da bomba de 2,7 $\mathrm{ml} / \mathrm{min}$.

As farinhas obtidas e a amostra in natura foram caracterizadas em triplicata quanto ao teor de umidade, $\mathrm{pH}$ e acidez total titulável conforme metodologia de IAL (2008). O teor de vitamina $C$ foi determinado por meio da reação do ácido ascórbico com o 2,6-diclorofenol indofenol (DCFI), conforme procedimento descrito por Oliveira (2010), e os resultados foram expressos em $\mathrm{mg} / 100 \mathrm{~g}$ de amostra (base seca).

A atividade de água das amostras foi determinada em Aqualab 3TE (Decagon, Devices USA) sob temperatura ambiente $\left(25^{\circ} \mathrm{C}\right)$. A cor foi avaliada por medida instrumental, utilizando espectrofotômetro MiniScan HunterLab XE Plus USA, no sistema de cor CieLab, obtendo-se as leituras de L* (luminosidade), $a^{*}$ (transição da cor verde - $a^{*}$ para 

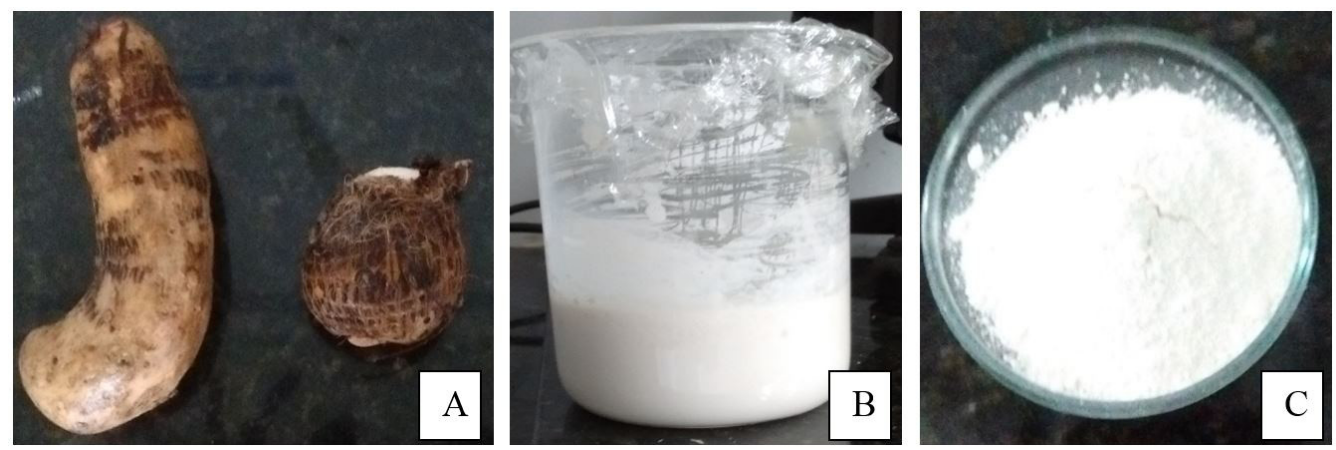

Figura 1. Taro in natura (A), extrato líquido após centrifugação $(B)$ e farinha de taro (C).

o vermelho $\left.+a^{*}\right)$ e $b^{*}$ (transição da cor azul $-b^{*}$ para a cor amarela $\left.+b^{*}\right)$.

Os compostos fenólicos totais foram estimados a partir do método de Folin e Ciocalteu, descrito por Waterhouse (2006), por meio da mistura de $75 \mu \mathrm{L}$ do suco filtrado do extrato com $2.050 \mu \mathrm{L}$ de água destilada, $125 \mu \mathrm{L}$ do reagente Folin-Ciocalteu e $250 \mu \mathrm{L}$ de carbonato de sódio 20\%, seguido de agitação e repouso por 5 minutos. A curva padrão foi preparada com ácido gálico, as leituras foram realizadas em espectrofotômetro a $765 \mathrm{~nm}$ em quatro pontos de concentrações (2, 4, 6 e $8 \mu \mathrm{g} / \mathrm{mL}$ ) e os resultados foram expressos em equivalente do ácido gálico (EAG) mg/100 g de massa seca.

A quantificação de flavonoides foi determinada conforme metodologia descrita por Woisky e Salatino (1998) e os resultados foram expressos em base seca e calculados com base em curva padrão obtida por etanol $95 \% / \mathrm{HCl} 1,5 \mathrm{~mol} / \mathrm{L}(85: 15)$ em quatro pontos de concentrações $(5,10,20$ e $40 \mu \mathrm{g} / \mathrm{mL})$.

Os resultados das análises foram submetidos a tratamento estatístico por meio de delineamento inteiramente casualizado com teste de comparação de médias, utilizando-se o software Assistat versão 7.7 beta (SILVA; AZEVEDO, 2009).

\section{Resultados e discussão}

A farinha de taro apresentou rendimento médio entre as temperaturas de secagem de 13\% com relação ao peso do extrato líquido. Na Tabela 1 encontram-se os dados da caracterização do taro in natura e secos, sob as temperaturas de $70^{\circ} \mathrm{C}, 80^{\circ} \mathrm{C}$ e $90^{\circ} \mathrm{C}$. Observou-se que a farinha de taro obtida sob as temperaturas de $80^{\circ} \mathrm{C}$ e $90^{\circ} \mathrm{C}$ não apresentaram diferença significativa entre si para os parâmetros de pH e acidez. Ao comparar os valores de $\mathrm{pH}(6,00,6,04,6,04)$ e acidez $(0,72$, $0,82,0,92)$ das farinhas com o pH $(6,36)$ e acidez $(0,37)$ do taro in natura, observou-se uma diminuição do $\mathrm{pH}$ das farinhas com consequente aumento da acidez, sendo estas características favoráveis para o armazenamento, por dificultarem o desenvolvimento de micro-organismos deteriorantes. A legislação brasileira para farinhas de mandioca (IAL, 1995) recomenda uma acidez total titulável $<3 \%$. Araújo et al. (2015), ao caracterizarem farinha de batata doce, observaram valores de acidez variando entre 0,64 e 0,52, de acordo com o aumento da temperatura de secagem. De acordo com estes autores, a acidez total titulável diminuiu com o aumento da temperatura, visto que os ácidos orgânicos se oxidam com a temperatura e se eleva a evaporação da água.

Quanto à umidade, as farinhas obtidas nas três temperaturas de atomização apresentaram características favoráveis ao armazenamento, garantindo segurança e estabilidade ao produto. Ladeira e Pena (2011), ao estudarem polvilho azedo obtido do cultivar Maranhense II, obtiveram $6,11 \%$ de umidade, aproximando-se do valor encontrado para a farinha obtida sob a temperatura de $80^{\circ} \mathrm{C}(6,16)$. Em relação à atividade de água, as farinhas apresentaram valores que thes asseguram estabilidade microbiológica $\left(a_{w}<0,6\right)$, de acordo com Fellows (2006).

Observou-se que, apesar de o maior percentual de vitamina $\mathrm{C}$ do taro in natura, este não diferiu estatisticamente do conteúdo de vitamina $\mathrm{C}$ encontrado nas farinhas obtidas por secagem a $80^{\circ} \mathrm{C}$ e $90^{\circ} \mathrm{C}$. Apesar de a vitamina $\mathrm{C}$ ser um composto termossensível, a sua concentração nas farinhas obtidas a $80^{\circ} \mathrm{C}$ e $90^{\circ} \mathrm{C}$ manteve-se praticamente inalterada com o processo de secagem, devido ao binômio tempo x temperatura, uma vez que a secagem em leito de jorro apresenta a vantagem de aumento da área de contato do produto durante a secagem, reduzindo sua exposição a temperaturas elevadas. Na secagem de $250 \mathrm{ml}$ de extrato líquido do taro, foram necessários tempos de $120 \mathrm{~min}, 95 \mathrm{~min}$ e $80 \mathrm{~min}$ para as temperaturas de $70^{\circ} \mathrm{C}$, $80^{\circ} \mathrm{C}$ e $90^{\circ} \mathrm{C}$, respectivamente.

A cor é um parâmetro importante para o produto quanto à aceitação do consumidor. A análise colorimétrica realizada no taro in natura e nas farinhas apresentou diferença significativa para a luminosidade $\left(\mathrm{L}^{*}\right)$, observando-se uma diminuição deste parâmetro nas farinhas quando comparado com o do tubérculo in natura. A escala do parâmetro $a^{*}$ varia do índice de saturação verde (-) ao vermelho (+), constando-se uma leve tendência de coloração para o verde. Os valores de $a^{*}$ do taro in natura e da farinha obtida 
Tabela 1. Caracterização físico-química do taro in natura e suas farinhas.

\begin{tabular}{|c|c|c|c|c|c|c|c|c|}
\hline Amostras & pH & $\begin{array}{l}\text { ATT } \\
(\%)\end{array}$ & $\begin{array}{l}\text { UM } \\
(\%)\end{array}$ & $A_{w}$ & $\begin{array}{c}\text { Vit. C } \\
(\mathrm{mg} / 100 \mathrm{~g})\end{array}$ & $L^{*}$ & $a^{*}$ & $\mathbf{b}^{*}$ \\
\hline In natura & $6,36 \pm 0,01^{a}$ & $0,37 \pm 0,02^{c}$ & $82,05 \pm 0,04^{a}$ & $0,99 \pm 0,01^{a}$ & $0,20 \pm 0,01^{a}$ & $79,91 \pm 0,16^{d}$ & $-0,50 \pm 0,07^{b}$ & $10,45+0,23^{b}$ \\
\hline $70^{\circ} \mathrm{C}$ & $6,00 \pm 0,01^{c}$ & $0,72 \pm 0,03^{b}$ & $6,81 \pm 0,17^{b}$ & $0,16 \pm 0,01^{b}$ & $0,05 \pm 0,01^{b}$ & $83,90 \pm 0,36^{c}$ & $-0,51 \pm 0,22^{b}$ & $12,46+0,61^{a}$ \\
\hline $80^{\circ} \mathrm{C}$ & $6,04 \pm 0,01^{b}$ & $0,86 \pm 0,04^{a}$ & $6,16 \pm 0,44^{b c}$ & $0,15 \pm 0,01^{b}$ & $0,15 \pm 0,02^{\mathrm{a}}$ & $85,17 \pm 0,16^{a}$ & $-0,94 \pm 0,09^{a}$ & $2,48+0,26^{a}$ \\
\hline $90^{\circ} \mathrm{C}$ & $6,04 \pm 0,01^{b}$ & $0,92 \pm 0,04^{a}$ & $5,61 \pm 0,27^{c}$ & $0,10 \pm 0,0^{c}$ & $0,17 \pm 0,02^{a}$ & $84,50 \pm 0,06^{b}$ & $-0,87 \pm 0,04^{a}$ & $3,13+0,10^{a}$ \\
\hline CV (\%) & 0,22 & 4,9 & 1,32 & 0,77 & 12,37 & 0,26 & 6,02 & 5,62 \\
\hline DMS (\%) & 0,03 & 0,09 & 0,86 & 0,42 & 0,04 & 2,36 & 0,23 & 1,16 \\
\hline
\end{tabular}

ATT - Acidez total titulável; UM - Umidade; A - Atividade de água. Médias seguidas pela mesma letra, na coluna, não diferem estatisticamente entre si pelo teste de Tukey $(p \leq 0,05)$. $n=3$.

Tabela 2. Conteúdo de compostos fenólicos totais e flavonoides (base seca) presentes no taro in natura e nas suas farinhas obtidas em leito de jorro sob as temperaturas de $70^{\circ} \mathrm{C}, 80^{\circ} \mathrm{C}$ e $90^{\circ} \mathrm{C}$.

\begin{tabular}{ccc} 
Amostras & $\begin{array}{c}\text { Compostos fenólicos } \\
(\mathbf{m g} / \mathbf{1 0 0} \mathbf{g} \text { b.s.) }\end{array}$ & $\begin{array}{c}\text { Flavonoides } \\
(\boldsymbol{\mu g} / \mathbf{1 0 0} \mathbf{g} \text { b.s.) }\end{array}$ \\
\hline In natura & $11,35 \pm 0,31^{\mathrm{a}}$ & $4,60 \pm 0,001^{\mathrm{a}}$ \\
$70^{\circ} \mathrm{C}$ & $5,60 \pm 0,01^{\mathrm{b}}$ & $3,40 \pm 0,001^{\mathrm{a}}$ \\
$80^{\circ} \mathrm{C}$ & $4,55 \pm 0,02^{\mathrm{c}}$ & $1,05 \pm 0,001^{\mathrm{b}}$ \\
$90^{\circ} \mathrm{C}$ & $4,42 \pm 0,09^{\mathrm{c}}$ & $4,30 \pm 0,001^{\mathrm{a}}$ \\
$\mathrm{CV}(\%)$ & 2,52 & 27,09 \\
$\mathrm{DMS}(\%)$ & 0,4252 & 0,024 \\
\hline
\end{tabular}

b.s.=base seca. Médias seguidas pela mesma letra, na coluna, não diferem estatisticamente entre si pelo teste de Tukey $(p \leq 0,05)$. $n=3$.

sob a temperatura de $70^{\circ} \mathrm{C}$ não apresentaram diferença significativa $(p<0,05)$, ambos diferindo estatisticamente dos valores de $a^{*}$ das farinhas obtidas sob as temperaturas de $80{ }^{\circ} \mathrm{C}$ e $90{ }^{\circ} \mathrm{C}$. Com relação ao parâmetro b*, todos os valores foram positivos, indicando que se encontram dentro da escala da intensidade de amarelo $\left(+b^{*}\right)$, não apresentando diferença significativa $(p \leq 0,05)$ para as diferentes temperaturas de secagem. A combinação dos valores dos parâmetros $L^{*}$, $a^{*}$ e $b^{*}$ das farinhas permitiram classificá-las como produto de coloração branca.

$\mathrm{Na}$ Tabela 2 encontram-se os valores de compostos fenólicos totais e flavonoides presentes no taro in natura e nas farinhas obtidas em leito de jorro sob as temperaturas de $70{ }^{\circ} \mathrm{C}, 80^{\circ} \mathrm{C}$ e $90^{\circ} \mathrm{C}$.

Na determinação de compostos fenólicos, notou-se que o taro in natura possui maior quantidade destes compostos, uma vez que a secagem promove a degradação de componentes termossensíveis dos alimentos.

A maior quantidade de compostos fenólicos nas farinhas estudadas foi encontrada naquela obtida a $70^{\circ} \mathrm{C}$, ressaltando a influência da temperatura sobre os compostos dos alimentos. Rocha (2012) relata que a farinha de polpa de batata da serra (Ipomoea convolvulácea L.) obtida sob temperatura de $50{ }^{\circ} \mathrm{C}$ por 3 horas, apresentou diminuição dos compostos fenólicos quando comparada com farinha obtida sob baixas temperaturas (liofilizada).

Quanto aos flavonoides, a maior quantidade também foi encontrada na amostra in natura, porém observou-se que a farinha de taro obtida em secagem a $90{ }^{\circ} \mathrm{C}$ apresentou maior valor de flavonoides quando comparada às farinhas obtidas sob as temperaturas de $70{ }^{\circ} \mathrm{C}$ e $80^{\circ} \mathrm{C}$, apresentando ainda redução de apenas $6,5 \%$ no conteúdo total de flavonoides quando comparada ao taro in natura. Casarin et al. (2016), ao estudarem o conteúdo de flavonoides em farinha de amora preta obtida em estufa, sob temperatura de $55^{\circ} \mathrm{C}$ por 16 horas, e comparar com o conteúdo de flavonoides presente na amora preta in natura, observaram uma diminuição de 9\% no conteúdo de flavonoides totais da farinha.

Apesar de os flavonoides serem compostos termossensíveis, estão sujeitos a uma maior degradação quando submetidos a altas temperaturas por tempo prolongado; assim, o menor teor de flavonoides observado na farinha obtida sob a temperatura de $80^{\circ} \mathrm{C}$ pode estar associado ao binômio tempo/temperatura. Fujita et al. (2013) também observaram que o aumento da temperatura de secagem foi pouco eficiente na degradação dos flavonoides em decorrência da diminuição do tempo de secagem, ao secarem polpa de camu-camu em leito de jorro.

\section{Conclusão}

Conclui-se que a farinha obtida pelo processo de leito de jorro, sob a temperatura de $90{ }^{\circ} \mathrm{C}$ apresentou os maiores conteúdos de vitamina $\mathrm{C}$ e flavonoides, porém a farinha obtida sob a temperatura de $70^{\circ} \mathrm{C}$ possui valores de compostos fenólicos superiores às demais temperaturas, desta forma, garantindo alterações mínimas no produto para este parâmetro. 
Efeito da temperatura sobre a composição físico-química e compostos bioativos de farinha de taro obtida em leito de jorro Castro, D. S. et al.

As amostras secadas nas temperaturas de $70^{\circ} \mathrm{C}$, $80{ }^{\circ} \mathrm{C}$ e $90^{\circ} \mathrm{C}$ possuem $\mathrm{pH}$ e acidez que irão garantir a vida de prateleira do produto, além da umidade reduzida, que são características favoráveis para prolongar a vida de prateleira de produtos alimentícios.

\section{Referencias}

ARAúJO, C. S. P.; ANDRADE, F. H. A.; GALDINO, P. O.; PINTO, M. S. C. Desidratação de batata-doce para produção de farinha. Agropecuária Científica do Semiárido, Campina Grande, v. 11, n. 4, p. 33-41, 2015.

BASSETTO, B. Z.; SAMULAK, R.; MISUGI, C.; BARANA, A.; ROSSO, N. Produção de biscoitos com resíduo do processamento de beterraba (beta vulgaris I.). Revista Verde de Agroecologia e Desenvolvimento Sustentável, Pombal, v. 8, n. 1, p. 139-145, 2013.

BEZERRA, C. V.; AMANTE, E. R.; OLIVEIRA, D. C.; RODRIGUES, A. M. C.; DA SILVA, L. H. M. Green banana (Musa cavendishii) flour obtained in spouted bed: effect of drying on physicochemical, functional and morphological characteristics of the starch. Industrial Crops and Products, Amsterdan, v. 41, p. 241-249, 2013. http://dx.doi.org/10.1016/j.indcrop.2012.04.035.

CASARIN, F.; MENDES, C. E.; LOPES, T. J.; MOURA, N. F. Planejamento experimental do processo de secagem da amorapreta (Rubus sp.) para a produção de farinha enriquecida com compostos bioativos. Brazilian Journal of Food Technology, Campinas, v. 19, n. 1, p. 1-9, 2016.

COSTA, R. G.; ANDREOLA, K.; MATTIETTO, R. A.; FARIA, L. G.; TARANTO, O. P. Effect of operating conditions on the yield and quality of açai (Euterpe) powder produced in spouted bed. LWT - Food Science and Technology, London, v. 64, n. 2, p. 1196-1203, 2015. http://dx.doi.org/10.1016/j.Iwt.2015.07.027.

FELLOWS, P. J. Tecnologia do processamento de alimentos: princípios e prática. Porto Alegre: Artmed, 2006. 602 p.

FUJITA, A.; BORGES, K.; CORREIA, R.; FRANCO, B. D. G. M.; GENOVESE, M. I. Impact of spouted bed drying on bioactive compounds, antimicrobial and antioxidant activities of commercial frozen pulp of camu-camu (Myrciaria dubia Mc. Vaugh). Food Research International, Oxford, v. 54, n. 1, p. 495-500, 2013. http://dx.doi.org/10.1016/j.foodres.2013.07.025.

INSTITUTO ADOLFO LUTZ - IAL. Métodos físico-químicos para análises de alimentos. 4. ed. São Paulo: Instituto Adolfo Lutz, 1995. $1020 \mathrm{p}$.
LADEIRA, T. M. S.; PENA, R. S. Propriedades físico-químicas e tecnológicas dos polvilhos azedos de três cultivares de mandioca. Alimentos e Nutrição, Araraquara, v. 22, n. 4, p. 631-640, 2011.

NASCIMENTO, W. F.; SIQUEIRA, M. V. B. M.; FERREIRA, A. B.; MING, L. C.; PERONI, N.; VEASEY, E. A. Distribution, management and diversity of the endangered Amerindian yam (Dioscorea trifida L.). Brazilian Journal of Biology = Revista Brasileira de Biologia, São Carlos, v. 75, n. 1, p. 104-113, 2015. http:// dx.doi.org/10.1590/1519-6984.08313.

OLIVEIRA, L. A. Manual de laboratório: análises físico-químicas de frutas e mandioca. Cruz das Almas: Embrapa Mandioca e Fruticultura, 2010. 248 p.

ROCHA, A. B. Caracterização bromatológica e avaliação de compostos bioativos presentes na batata da serra (ipomoea convolvulácea I.) produzida na chapada diamantina-BA. 2012. 111 f. Dissertação (Mestrado em Ciência de Alimentos)Universidade Fedeal da Bahia, Salvador, 2012.

SANTOS, E. S.; CEREDA, M. P.; PEDRALLI, G.; PUIATTI, M. Dominações populares das espécies Dioscorea e Colocasia no Brasil. Revista Tecnologia \& Ciência Agropecuária, João Pessoa, v. 1, n. 1, p. 37-41, 2007.

SILVA, F. A. S.; AZEVEDO, C. A. V. Principal components analysis in the software assistat-statistical attendance. In: WORLD CONGRESS ON COMPUTERS IN AGRICULTURE, 7., 2009, Reno. Proceedings... Reno: American Society of Agricultural and Biological Engineers, 2009.

SOUZA, D. S.; SOUZA, J. D. R. P.; COUTINHO, J. P.; FERRÃO, S. P. B.; SOUZA, T. S.; SILVA, A. A. Elaboração de farinha instantânea a partir da polpa de fruta-pão (Artocarpus altilis). Ciência Rural, Santa Maria, v. 42, n. 6, p. 1123-1129, 2012. http://dx.doi.org/10.1590/S0103-84782012005000026.

TEIXEIRA, A. P.; OLIVEIRA, I. M. A.; LIMA, E. S.; MATSUURA, T. The use of purple yam (Dioscorea trifida) as a health-promoting ingredient in bread making. Journal of Research in Biology, Tirunelveli, v. 3, n. 1, p. 747-758, 2013.

WATERHOUSE, A. Oxidation of wine phenolics: a critical evaluation and hypotheses. American Journal of Enology and Viticulture, Davis, v. 57, p. 356-357, 2006.

WOISKY, R. G.; SALATINO, A. Analysis of pró-polis: some parameters and procedures for chemical quality control. Journal Apicultural Research, Groombridge, v. 37, n. 2, p. 99-105, 1998. 